\title{
Acquired Colovesical Fistula
}

\author{
Kelly Ratheal MD
}

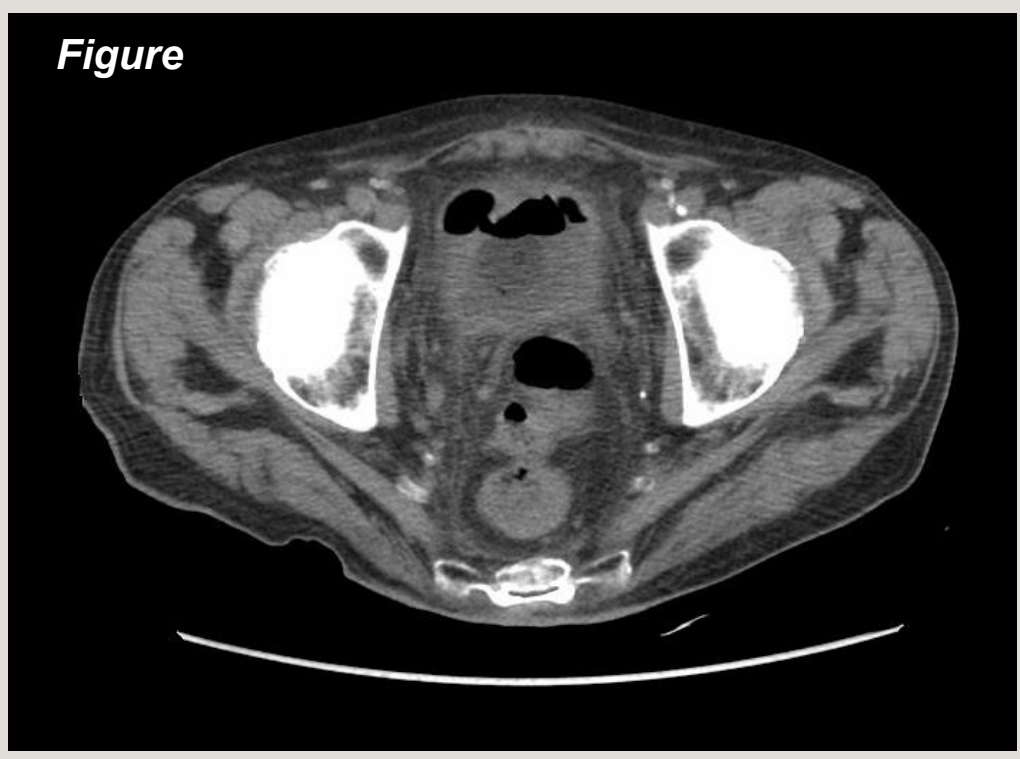

\section{CASE REPORT}

An 81-year-old man was brought to the emergency department with a two day history of altered mental status, decreased oral intake, diarrhea, and fever. Reportedly, he had been unable to care for himself for several days prior to his presentation. The patient appeared lethargic, malnourished, and unkempt. On examination, he was tachypneic, tachycardic, and febrile and had a large sacral decubitus ulcer. He was diagnosed with acute severe sepsis likely secondary to urinary tract infection and the sacral ulcer. A stool panel ruled out infectious diarrhea. Guarding on abdominal examination at presentation prompted computed tomography (CT) of the abdomen, which

Corresponding author: Kelly Ratheal MD

Contact Information: Kelly.Ratheal@ttuhsc.edu DOI: 10.12746/swrccc 2016.0413.172 showed rectosigmoid diverticulosis without evidence of diverticulitis and some thickening or edema of the superior urinary bladder (which contained a catheter) possibly representing inflammation or cystitis. Upon further review of these images it was determined that the patient had a colovesical fistula. An ultrasound of the abdomen revealed a Foley catheter in the bladder and a one centimeter echogenic lesion on the posterior bladder wall consistent with a clot or mass. Cystoscopy and a retrograde cystogram were not performed, since the patient was not stable enough to undergo these procedures.

\section{Discussion}

Enterovesical fistula is a rare finding. This term describes a connection between the bladder 
and either the small or large intestine. The most common etiology of these fistulae is inflammatory bowel disease, specifically diverticulitis, in which a fistula is formed by direct extension of a ruptured diverticulum or erosion of a peridiverticular abscess into the bladder. ${ }^{1}$ The relative risk for developing enterovesical fistula in the presence of diverticular disease is 1 to 4 percent. ${ }^{1}$ Cancer, particularly of the bowel, is the second most common cause (10-20\% of cases) of an enterovesical fistula. ${ }^{1}$ Although infrequent, squamous cell carcinoma of the bladder and lymphoma may also cause these fistulae. Crohn's disease is the third leading etiology (5-7\%). Other etiologies include chemotherapy and/or radiation complications, urogenital malignancies, and procedure related complications. Foley catheterization has been reported as a rare cause of enterovesical fistula. ${ }^{2}$ This patient did not have a Foley catheter in place prior to his admission. The Foley catheter was placed after his arrival to the emergency room and before the CT abdomen. Consequently, traumatic fistula formation is possible but very unlikely. The patient did have rectosigmoid diverticulosis seen on his CT scan of the abdomen. A ruptured or eroded diverticulum is the most likely cause of his fistula.

Enterovesical fistulae commonly present as fecaluria, pneumaturia, and recurrent urinary tract infection. ${ }^{3}$ Identification of enterovesical fistulae is difficult, and patients may go for months without diagnosis. Additionally, reports have been made of patients having air in the bladder as an incidental finding upon imaging for other various reasons prior to the diagnosis of enterovesical fistula. ${ }^{4}$ Diagnosis of enterovesical fistula can be made several ways. CT scan has $70 \%$ specificity and nuclear cystography $80 \%$ specificity. Cystoscopy, while useful in identifying potential bladder lesions, misses the fistula $54-65 \%$ of the time. The poppy seed test, however, is $100 \%$ specif-

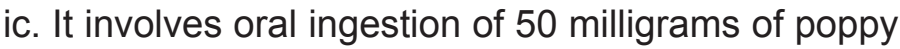
seeds mixed into liquid, which remain undigested as they pass through the gastrointestinal tract. If they appear in the urine, usually within 48 hours, enterovesical fistula is confirmed. ${ }^{1}$ The poppy seed test is very specific, but a CT scan is the modality of choice due to its ability to give additional information regarding the anatomy adjacent to the fistula. Key findings on CT that indicate enterovesical fistula are air in the bladder, oral contrast in the bladder, presence of colonic diverticula, and bladder wall thickening next to a segment of thickened intestine. Air in the bladder is pathognomonic; however false positives may occur after recent bladder instrumentation and during active urinary tract infection with a gas-forming organism. ${ }^{1}$ This patient did have air in the bladder on CT scan (Figure). He also had colonic diverticula and a thickened portion of the bladder which support colovesical fistula as the diagnosis. Obtaining history about recurrent UTI and pneumaturia was difficult due to his altered mental status and poor condition.

Treatment of enterovesical fistula requires surgery in most cases, but it can be managed conservatively. ${ }^{5}$ The surgical approach usually involves bowel resection with primary anastomosis and excision of the bladder lesion, if carcinoma is present, with subsequent bladder repair. ${ }^{1}$

\author{
Author affiliations : Kelly Ratheal is a resident in the Depart- \\ ment of Internal Medicine at Texas Tech University Health \\ Sciences Center in Lubbock, TX. \\ Submitted: $1 / 7 / 2016$ \\ Accepted: $1 / 10 / 2016$ \\ Reviewers: Eman Attaya MD \\ Conflict of Interest: None \\ Published electronically: 1/15/2016
}

\section{References}

1. Golabek T, Szymanska A, Szopinski T, et al. Enterovesical fistulae: aetiology, imaging, and management. Gastroenterol Res Pract 2013; 2013: 617967, 8 pages. Epub 2013 Nov 21

2. Hawary A, Clarke L, Taylor A, et al. Enterovesical fistula: a rare complication of urethral catheterization. Adv Urol 2009; 591204. Epub 2009 Aug 3.

3. Law WL, Chu SM. An unusual enterovesical fistula. Am J Surgery 2008; 195 (6): 814-15.

4. Kao PF, Ting WC, Hsiao PC, et al. Dynamic FDG PET/CT imaging with diuresis demonstrates an enterovesical fistula in a lymphoma patient with repeated colon diverticulitis. Clin Nucl Med 2013; 38 (4): 272-5.

5. Scozzari G, Arezzo A, Morino M. Enterovesical fistulas: diagnosis and management. Tech Coloproctol 2010; 14 (4): 293-300. 